Article

\title{
Nonlinear Whole Seismology, Topological Seismology, Magni- tude-Period Formula of Earthquakes and Their Predictions
}

\author{
Yi-Fang Chang
}

How to cite this paper: Chang, Y. F. (2021). Nonlinear Whole Seismology, Topological Seismology, Magnitude-Period Formula of Earthquakes and Their Predictions. World Journal of Geomatics and Geosciences, 1(1), 1-10. DOI:

10.31586/wjgg.2021.010101. Retrieved from https://www.scipublications.com/journal/index.php/wjgg/article/view/46
Received: June 2, 2021

Accepted: July 16, 2021

Published: July 17, 2021

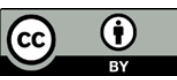

Copyright: (c) 2021 by the authors. Submitted for possible open access publication under the terms and conditions of the Creative Commons Attribution (CC BY) license (http://creativecommons.org/license s/by/4.0/).
Department of Physics, Yunnan University, Kunming 650091, China

* Correspondence: yifangch@sina.com

\begin{abstract}
First, we propose the nonlinear whole seismology and its three basic laws. Next, based on the nonlinear equations of fluid dynamics in Earth's crust, we obtain a chaos equation, in which chaos corresponds to the earthquake, and shows complexity on seismology. But, combining the Carlson-Langer model and the Gutenberg-Richter relation, a simplified nonlinear solution and corresponding magnitude-period formula of earthquakes may be derived approximately. Further, we research the topological seismology. From these theories some predictions can be calculated quantitatively and are already tested. Combining the Lorenz nonlinear model, we may discuss the earthquake migration to and fro. Finally, if various modern scientific instruments, different scientific theories and some paranormal ways for earthquake are combined each other, the accuracy of multilevel prediction will be increased.
\end{abstract}

Keywords: Seismology, Nonlinear, Topology, Earthquake, Chaos, Fluid Dynamics, Prediction

\section{Introduction}

It is recognized that the earthquakes are very complex nonlinear phenomena, and many theories and some phenomenological descriptions have been proposed, such as fractals, and propagation and interaction on the seismic wave in the nonlinear media [13]. Carlson, Langer, et al. [4-8], presented the Burridge-Knopoff block-and-spring model of an earthquake fault, and discussed basic properties, predictability and so on.

Based on the Gutenberg-Richter (GR) relation, we proposed the magnitude-period formula of the earthquake $[9,10]$ :

$$
T=10^{\left(a_{0}-a\right)-\left(b_{0} M_{0}-b M\right)} T_{0}
$$

and its simplified formula:

$$
T=10^{-b\left(M_{0}-M\right)} T_{0}
$$

Topology is the mathematical study on properties of space preserved under continuous deformations including stretching and bending, but not tearing or gluing. It includes connectedness, continuity and boundary. Topology is widely applied to various aspects $[11,12]$. In this paper, we propose the nonlinear whole seismology, topological seismology and their applications.

\section{Nonlinear Whole Seismology and Its Basic Laws}

Based on the most basic features of modern seismology and combined geological structure and seismic mechanism, we propose the nonlinear whole seismology and its three basic laws: 
First law: Seismology as whole is a complex huge system of violent crustal movement.

Second law: Various interactions in seismology all are nonlinear. Corresponding equations all are nonlinear, and possess chaos and fractals, etc. Different magnitudes $\mathrm{M}$ possess fractals with self-similarity.

Third law: Earth and crust as environment are closely related to earthquakes. They are regarded as boundary conditions of the system, which and the seismological systems have often various nonlinear relations.

Two main characters of seismology are nonlinearity and whole. Three laws are a whole: Because of complexity, the description of seismological systems must apply the nonlinear theory with the interaction terms. Its future development is the nonlinear whole earth science.

We researched possible relations on earthquakes and various cycles of crustal movement, terrestrial magnetic field, Earthrotation, change in climate, and sunspot [9] which is related to nonlinear Earth-Sun physics. They show also whole and complexity.

\section{Nonlinear Geodynamics and Seismology}

Based on general geodynamics [13], and combines the Burridge-Knopoff block-andspring model of an earthquake fault presented by Carlson, Langer, et al. [4-8], we proposed that the fundamental nonlinear equations of fluid mechanics in Earth's crust with momentum conservation are:

$$
\rho \frac{D V}{D t}=\rho\left(\frac{\partial V}{\partial t}+V \nabla V\right)=F_{0}-\operatorname{grad} p+f .
$$

Here the frictional force for Newtonian fluid is:

$$
f=\eta \Delta v+\left(\zeta+\frac{\eta}{3}\right) \operatorname{graddiv} V .
$$

Usual solutions of the nonlinear fluid mechanics are very difficult.

In order to simplify the above case, assume that $F_{0}=0$, the pressure gradient gradp $=C^{\prime \prime}$ is independent of the velocity $\mathrm{V}$, so Eq. (3) turns into

$$
\frac{\partial V}{\partial t}+V \nabla V=-\frac{C^{\prime \prime}}{\rho}+\frac{\eta}{\rho} \Delta V+\frac{3 \zeta+\eta}{\rho} \text { graddiv } V .
$$

This is a nonlinear partial differential equation of the velocity $\mathrm{V}$. Using a similar way for the soliton solution, let $\xi=\alpha(x+y+z-u t)$, so Eq. (5) becomes an ordinary differential equation:

$$
(v-u) v^{\prime}=-\frac{C^{\prime \prime}}{\rho \alpha}+\alpha b v^{\prime \prime} .
$$

Here $b=(4 \eta / 3+\zeta) / \rho$. If $C^{\prime \prime}=0$, the equation is:

$$
\frac{d v}{d \xi}=\frac{1}{2 \alpha b}\left(v^{2}-2 u v\right)+C^{\prime}
$$

This solution is: 


$$
v=u+b \sqrt{B} c t h\left(-\frac{1}{2} \sqrt{B} \xi+C\right),
$$

for $(u / \alpha b)^{2}-\left(2 C^{\prime} / \alpha b\right)=-B^{-1}<0$. When $C^{\prime}=0$, the equation is:

$$
\frac{d v}{d \xi}=\frac{v}{2 \alpha b}(v-2 u)
$$

whose solution is:

$$
v=\frac{2 u}{1-\exp [(u \xi / \alpha b)+C]} .
$$

Therefore, the kinetic energy density is:

$$
\varepsilon=\frac{1}{2} \rho v^{2}=\frac{2 \rho u^{2}}{\{1-\exp [(u(r-u t) / b)+C]\}^{2}} .
$$

When $r=0$ and $t=0, \varepsilon=\left(2 \rho u^{2}\right) /\left(1-e^{c}\right)^{2}$. Let the integral constant $C>0$, the larger the region is, the smaller the energy density $\varepsilon$ is at the same time t; the larger $\varepsilon$ becomes as time $\mathrm{t}$ increasing for the same region $r$. If $t_{0}=(r / u)+c(4 \eta+3 \zeta) / 3 \rho u^{2}, \varepsilon=\infty$. It is an unreachable value. When momentumenergy are transported and accumulated, and once the accumulation of energy $(\varepsilon)$ with time at the same region excesses a faulting threshold value $\left(\varepsilon_{0}\right)$ of rock in the locality, earthquake will occur with the energy releases. From this we may decide to have some relations among the geological structure, the faulting threshold value and the integral constant C.

The equation (9) corresponds to a difference equation:

$$
v_{n+1}=\frac{1}{2 \alpha b} v_{n}\left(v_{n}-2 u\right) .
$$

Using a substitution $v=u(1-u x / 2 \alpha b)$, Eq.(12) becomes:

$$
x_{n+1}=1-\frac{u^{4}}{(2 \alpha b)^{2}} x_{n}^{2} .
$$

It is a well-known nonlinear chaos equation. For momentum the control parameter is:

$$
\lambda=\left(\frac{u^{2}}{2 \alpha b}\right)^{2}=\frac{9 u^{4} \rho^{2}}{4 \alpha^{2}(4 \eta+3 \xi)^{2}}>0 .
$$

This determines the branch-chaos. If our consideration is in more detail, $\lambda$ will be more complex. Various similar results may be approximately derived from the energy conservation equations.

This fluid layer may be a general asthenosphere. As an example, the matter density is $\rho \approx 4 \mathrm{~g} / \mathrm{cm}^{3}$ and $\eta \approx 10^{22} \mathrm{p}$. Let $\alpha \approx 1, t_{0}$ is enough long, $\lambda<<1$, so the usual 
crust is stable. But, if the asthenosphere consists of solid, fluid, gas and plasma, $\eta$ will decrease very quickly. For a special asthenosphere, when $\lambda \geq 0.75$, the bifurcation will appear, perhaps it corresponds to the momentum migration between a couple of places. If $\eta$ decreases suddenly at a fault, $\lambda=1.401155 \ldots .$. , it infers a chaos will appear, and corresponding earthquake occurs. In fact, earthquake will occur as the shorter the series of calm time, which is consistent with a branch-chaos process. This could be a basis of the renormalization schemes [9], which research some seismic activity prior to the main earthquake.

Some factors may cause earthquake, for example, volcanism, reservoir, underground nuclear explosion and so on [15-17], which are merely boundary or initial conditions. But, because the seismic system obeys the nonlinear equation, they are extremely sensitive to the initial conditions.

It is known that earthquakes are very complex nonlinear phenomena. At present the nonlinear seismology is an important direction of the development. A main character of nonlinearity is chaos. In Webster's Third Dictionary "chaos" possesses these meanings of chasm, gulf and abyss. These are namely results of earthquake. Turcotte discussed generally fractals and chaos in geology and geophysics [2]. Levin researched nonlinear oscillating structures in the earthquake and seaquake dynamics [3].

The earthquake corresponds to chaos, which shows complexity on seismology, and is useful for the explanation of seismic essence, and casts a fatal shadow in an earthquake exact prediction. However, the possible periodicity in earthquakes may be determined according to the approximate results from a simplified model.

\section{Magnitude-Period Formula of Earthquake}

For the forecasts of earthquakes, Kiremidjian, et al. [18] presented a stochastic slippredictable model based on Markov renewal theory for earthquake occurrences. Harris, et al., discussed changes and influence of static stress on earthquake in southern California [19-21]. Borodich [14] described some renormalization schemes for earthquake prediction, which can be reduced to a power-law or the log-periodic approximation of the regional seismic-activity data. Marzocchi, et al. [22] provided insights that might contribute to better formally defining the earthquake-forecasting problem, both in setting up and in testing the validity of the forecasting model, and found that the forecasting capability of these algorithms is very likely significantly overestimated. Helmstetter, et al. [23] developed a time-independent forecast for southern California by smoothing the locations of magnitude 2 and larger earthquakes, and using small $m \geq 2$ earthquakes gives a reasonably good prediction of $m \geq 5$ earthquakes.

We combine the Carlson-Langer model [4-8], the movable asthenosphere corresponds to the surface, and the faulting rock layer corresponds to the block. From this the Gutenberg-Richter relation and the same results may be obtained, while in such case the masses of blocks are usually different, and the velocities may be changeable, especially at the fissure. Further, from some formulas of the Carlson-Langer model, the magnitudeperiod formula [24] may be derived. In the model, the magnitude $M$ is be defined to be the natural logarithm of the earthquake moment $H$,

$$
M=k \ln H .
$$

Let the moment

$$
H=C \int_{T_{0}}^{T_{0}+T} \dot{X} d t
$$

between two times $T_{0}$ and $T_{0}+T$. In the model, the frictional force is 


$$
F(\dot{X})=\frac{F_{0}}{1+\dot{X}}
$$

which is also a result in a simple mechanical model for earthquake dynamics [25]. In present paper the frictional force is

$$
\begin{aligned}
& F=\eta \Delta V+\left(\frac{\eta}{3}+\zeta\right) \text { graddiv } V \approx\left(\frac{4 \eta}{3}+\zeta\right) v^{\prime \prime} \\
& =\frac{2 u^{3}}{(\alpha b)^{2}} \exp \left(\frac{u \xi}{\alpha b}+c\right)\left[1+\exp \left(\frac{u \xi}{\alpha b}+c\right)\right] /\left[1-\exp \left(\frac{u \xi}{\alpha b}+c\right)\right]^{3} .
\end{aligned}
$$

If both forces $\mathrm{F}$ are equal,

$$
\dot{X}=F_{0} \frac{(\alpha b)^{2}}{2 u^{3}} \frac{[1-\exp (u \xi / \alpha b+c)]^{3}}{\exp (u \xi / \alpha b+c)[1+\exp (u \xi / \alpha b+c)]}-1 .
$$

Only the time variable is related, so $\xi \approx-\alpha u t+c$, which is replaced to Eq.(16), and let $d=u^{2} / b$,

$$
\begin{gathered}
H_{0}=C\left[F_{0} \frac{(\alpha b)^{2}}{2 u^{3}}\left(4 \ln \left|1+e^{d t+c}\right|-e^{d t+c}-e^{-d t-c}\right)-t\right]_{T_{0}}^{T+T_{0}} \\
\approx C\left[F_{0} \frac{(\alpha b)^{2}}{2 u^{3}}(2+4 d t+4 c)-t\right]_{T_{0}}^{T+T_{0}}=C\left(\frac{2 \alpha^{2} b F_{0}}{u}-1\right) T . \\
M=k \ln C+k \ln \left(\frac{2 \alpha^{2} b F_{0}}{u}-1\right) T .
\end{gathered}
$$

Let $k \ln C=M_{0}$ and $\left(2 \alpha^{2} b F_{0} / u\right)-1=1 / T_{0}$, so

$$
M=M_{0}+k \ln \frac{T}{T_{0}}=M_{0}+2.3026 k \lg \frac{T}{T_{0}}=M_{0}+\frac{1}{b} \lg \left(\frac{T}{T_{0}}\right) .
$$

It is namely a simplified magnitude-period formula of the earthquake $[9,10,24]$ :

$$
T=10^{-b\left(M_{0}-M\right)} T_{0}
$$

It is namely my formula (2). Its general formula is (1). In this case, $\mathrm{b}$ is a tectonic parameter in the Gutenberg-Richter relation. The fractal characteristics of earthquakes show the fractal dimension $D=2 b$ [26]. If $k=1, b=0.43429$ agrees with the earthquake belt of the longer period of earthquakes, whose $b=0.4-0.6$. If $k=0.5-0.333, b=0.86858-1.3029$ [27]. The longer the period $\mathrm{T}$ of earthquakes is, the less the seismic number $\mathrm{N}$ is. The formula (23) and the Tsubokawa-Whitcomb-Scholz relation $[28,29]$

$$
\log T=a M-b,
$$

between duration of crustal movement and magnitude of earthquake, have the same form and but different meanings and signs of parameters. The solutions of the one dimensional partial differential equations for Carlson-Langer model have the form [21]: 


$$
U(x, t) \approx-(1-\varepsilon) \sin \left[\frac{x-v t}{\sqrt{v^{2}-\xi^{2}}}\right],
$$

which implies a period.

Almost all formulas of the forecasts on earthquakes are based on the Gutenberg-Richter relation, which can estimate the average rate of earthquakes. We assume that $\mathrm{T}$ and $\mathrm{N}$ are inversely proportional in a first-order approximation, so the magnitude-period formula (2) of earthquakes may be derived from the Gutenberg-Richter relation [9]. But, the formula is built on the nonlinear dynamical theory, so it may be researched widely.

\section{Seismological Topology}

Based on the chaos equation (13) of earthquakes, they may have periods two, four, eight and so on, for example, period is two 0.5130 and 0.7995 , or four $0.38,0.83,0.88$ and 0.50 , etc. This type of earthquakes may use the Fourier series:

$$
f(x)=a_{0} / 2+\sum_{n=1}^{\infty}\left(a_{n} \cos n x+b_{n} \sin n x\right) .
$$

It is the superposition of various periods, and whose formula

$$
T=\omega x=(n \pi / l) x .
$$

It may replace into the magnitude-period formula (2). According to the Landau equation of the turbulence:

$$
\frac{d A}{d t}=\sigma A-\frac{l}{2} A^{3} .
$$

Its two simple solutions are: (1). $\mathrm{A}=0(\mathrm{r}=0)$ is zero-earthquake; (2). The limit cycle of the radius $r=\sqrt{2 \sigma / l}$ is the periodic earthquakes.

An earthquake field may be proposed phenomenally. Generally, the nonlinear differential equation has the stable multi-solutions, so that the nonlinear earthquakes have the multi-periods. Probably, many periods reach finally chaos, which obtains the strange attractor and determines the central point of earthquake, earthquake region and fractal. And $b$ in Eq.(2) and a self-similar period may be evaluated from the fractal dimension D. The three dimensional qualitative analysis theory of nonlinear equation may determine the longitude-latitude-depth, or the longitude-latitude-time. While a stable singularity shows that this point will be necessarily scene of earthquake. Attractor may be applied to a formation of earthquake.

The continuous fluid may form a topological space, and it is a flow topology. The dynamical system of chaos may be defined by topology [12], such a chaotic meaning of earthquake contacts naturally the topology of earthquake. Further, we research the topological seismology, in which 1). Earthquake is topology torn by tension. 2). The fixed point is an earthquake region. 3). For 2-, 3-, 4-dimensional topology, earthquake point corresponds to a cut point (a sensitive point), and may develop to a small region, which contacts network, for which the self-similarity and fractal exist possibly. 4). Different networks form a series of $p_{0}, p_{1}, p_{2}, \ldots p_{k} \ldots$, which and $\mathrm{k}$ ( $\mathrm{k}$ is a value of the annular handle of a spherical surface) define a frequency of earthquake. Zero-earthquake and disc-sphere are homeomorphic. Here $\mathrm{k}$ may be the 3-dimensional invariant, or the 2-dimensional $\mathrm{k}(\mathrm{t})$. From this we introduce the Euler characteristic of a curved surface Q: 


$$
\chi(\mathrm{Q})=\mathrm{V} \text { (vertex)-E(edges) }+\mathrm{F}(\text { number of polygon), }
$$

which is a topological invariant. For the 2- and 3-dimensional spaces, the simplest cases are $\chi=1$ and 2. If $\mathrm{V}(\mathrm{t})$ is a function of time, $\mathrm{E}(\mathrm{t})$ and $\mathrm{F}(\mathrm{t})$ will be relative to time $\mathrm{t}$, but $\chi$ should be invariant. 5). A curved surface with k-holes may be projected to a plane with hole $(\leqslant k)$.

The equations of fluid dynamics on geodynamics combine the phase transition point $\lambda$ and Lorenz model, etc., we can research the identification scales on stability of the earth's crust and on occurred earthquake. These may introduce the Lyapunov exponent.

The topological seismology shows seismology as a whole. Its development is the topological earth science [30].

Moreover, we propose that research of earthquake may be: (1). The space-time point of earthquake is described by computer, and further computer simulation and programs of earthquakes. (2). Usual earthquake is stochastic, and corresponds to chaos. But, there is the self-similarity (for magnitude, etc.) and fractal. (3). Carlson-Langer model [5-8] and many nonlinear equations derive various soliton solutions, which may describe the earthquake wave. (4). When $b$ in Eq.(2) develops to a function of time $b(t)$, the period $T$ is related to time $\mathrm{t}$, and $\mathrm{N}=\mathrm{C} / \mathrm{T}$ ( $\mathrm{C}$ is a function). (5). The sink and source by a qualitative analysis theory of nonlinear earthquake equations should be the center earthquake. (6). For a region, we may describe the magnitude-time, the space-time-magnitude, and the general 4dimensional longitude-latitude-time-magnitude figure.

\section{Predictions of Earthquakes}

According to the magnitude-period formula (2), so long as one supposes a possible period of earthquakes, for example, the period 34 year in California, other periods can be calculated approximately. Bi and Yuan proposed a periodic scale 250 year for change of weather in China from various aspects. We extended the periodic scale $T_{0}=250$ year to a period of earthquakes for $M_{0}=7$, and results are consistent with many known earthquakes in China [9]. From this the quantitative calculations can be given. The period $\mathrm{T}$ that corresponds to the magnitude $\mathrm{M}$ will depend on $\mathrm{b}$ only, if the scale is determined. Either evaluation of $\mathrm{T}$ from $\mathrm{b}$ or evaluation of $\mathrm{b}$ from $\mathrm{T}$ agrees approximately with the facts. It is a simplified and calculable model.

On earthquakes in California Smith, et al., searched strain adjustments associated with earthquakes in southern California [31]. Kovach researched source mechanisms for Wilmington oil field, California, subsidence earthquakes [32]. Mavko, et al., discussed effects of the 1983 Coalinga, California, earthquake on creep along the San Andreas fault [33]. Oppenheimer, et al., obtained the fault plane solutions for the 1984 Morgan Hill, California, earthquake sequence as evidence for the state of stress on the Calaveras fault [34]. Reasenberg, et al., discussed earthquake hazard after a main shock in California [35]. Jaumé, et al., researched change in the state of stress on the southern San Andreas fault resulting from the California earthquake sequence of April to June 1992 [36]. Stein, et al., searched stress triggering of the $1994 \mathrm{M}=6.7$ Northridge, California, earthquake by its predecessors [37]. Dodge, et al., observed detail on California foreshock sequences as implications for the earthquake initiation process [38]. Deng and Sykes studied evolution of the stress field in southern California and triggering of moderate-size earthquakes for a 200-year perspective [39], and discussed stress evolution in southern California and triggering of moderate-, small-, and micro-size earthquakes [40]. Rhoades applied the EEPAS model to forecasting earthquakes of moderate magnitude in Southern California [41]. Based on the basic principle of general model of forecasts, Gerstenberger, et al., discussed real-time forecasts of tomorrow's earthquakes in California [42]. Ebel, et al., searched the non-Poissonian earthquake clustering and the hidden Markov model as bases for earthquake forecasting in California [43]. 
From (1) and (2) we may predict quantitatively earthquakes. California is a multiearthquake area, but bigger ones are still not more. When $b=0.86868$, various periods are $\mathrm{T}=33.83,4.579,0.6197$ year for $\mathrm{M}=7,6,5$, respectively, here $\mathrm{T}=33.83$ year agrees with the periods of earthquakes in California. From 2004 to 2017, according to the formula (2) we predicted future earthquakes will take place in 2009, 2014 and 2019, and large earthquake of 2019 as intersection of two series of earthquakes will be more possible [44,24,45]. Then earthquakes (M=6.9) occurred on 3 August of 2009, and $M=6$ on 24 August of 2014. In 2019 California occurred two bigger earthquakes on 4 July $\mathrm{M}=6.4$ and 5 July $\mathrm{M}=7.1$. This shows that earthquakes may be predicted initially, and be calculated simply. For example, a new series of predictions on period 33.83 years for $\mathrm{M}=7$ in California region will be:

\begin{tabular}{|c|c|c|c|}
\hline Year & 2019 July & 2053 & 2086 \\
\hline Magnitude & 7.1 & & \\
\hline
\end{tabular}

For the period $\mathrm{T}=4.579$ year of $\mathrm{M}=6$, the corresponding earthquakes in California will be:

\begin{tabular}{|c|c|c|c|c|c|c|c|c|}
\hline Year & July 2019 & 2025 & 2029 & 2034 & 2039 & 2044 & 2049 & 2053 \\
\hline Magnitude & 6.4 & & & & & & & \\
\hline
\end{tabular}

Here 2053 will be new intersection of two series of earthquakes.

Further, based on the Lorenz nonlinear model with two "wings" [46], we researched the earthquake migration to and fro, and two or a few time series on earthquakes in the same region $[47,48,24,45]$.

Ramos, et al. [49], introduced a modification of the Olami-Feder-Christensen earthquake model in order to improve the Burridge-Knopoff mechanical model. Dynamical disorder is added to the thresholds following a narrow distribution. They found quasiperiodic behavior in the avalanche time series with a period proportional to the degree of dissipation of the system. Periodicity is not as robust as criticality when the threshold force distribution widens, or when an increasing noise is introduced in the values of the dissipation.

Faces to huge natural suffering, if humanity could give up some conservative viewpoints, earthquake predictions should be combined different methods [44,24,45]: various modern scientific instruments, different scientific theories (including the magnitude-period formula of earthquakes, topological method, and so on), and some paranormal ways, which include special functions and animals and plants, etc [50,51]. Such a network of multilevel earthquake prediction will be able to be developed, and will be able to increase the accuracy of prediction.

\section{Reference}

[1] Y.Y. Kagan, and L. Knopoff, Stochastic synthesis of earthquake catalogs. J. Geophys.Res. 1981, 86: $2853-2862$.

[2] D.L. Turcotte, Fractals and Chaos in Geology, and Geophysics. Cambridge University Press 1992.

[3] B.W. Levin. Nonlinear oscillating structures in the earthquake and seaquake dynamics. Chaos. 1996, 6(3):405-413.

[4] J.M. Carlson and J.S. Langer. Properties of earthquakes generated by fault dynamics. Phys. Rev. Lett. 1989, 62, $2632-2635$.

[5] J.M. Carlson and J.S. Langer. Mechanical model of an earthquake fault. Phys.Rev. 1989, A40,6470-6484.

[6] J.M. Carlson, J.S. Langer, B.E. Shaw and C. Tang. Intrinsic properties of a Burridge-Knopoff model of an earthquake fault. Phys.Rev. 1991, A44,884-897.

[7] J.M. Carlson. Two-dimensional model of a fault. Phys.Rev. 1991, A44,6226-6232.

[8] J.M. Carlson, J.S. Langer and B.E. Shaw. Dynamics of earthquake faults. Rev.Mod.Phys. 1994,66, 657-671.

[9] Yi-Fang Chang, Periodicity of change in Earth-Sun and research of period of earthquake. In: New Research of Particle Physics and Relativity. Yunnan Science Technology Press. 1989. p259- 269. Phys.Abst. 93,1371, 30443(1990).

[10] Yi-Fang Chang, Research of nonlinear dynamical system on earthquake. Exploration of Nature (China). 1997,16(3):51-55.

[11] S. Lefschetz, Applications of Algebraic Topology. Springer-Verlag. 1975.

[12] C. Adams and R. Franzosa, Introduction to Topology: Pure and Applied. Prentice Hall. 2007. 
[13] D.L. Turcotte, and G. Schubert, Geodynamics. John Wiley and Sons. 1982.

[14] M. Borodich. Renormalization schemes for earthquake prediction Geophys.J.Int. 1997, 131,171-178.

[15] R.M. Hamilton, B.E. Smith, F.G. Fischer and P.J. Papanek. Earthquakes caused by underground nuclear explosions on Pahute Mesa, Nevada Test Site. Bull.Seismol.Soc.Am. 1972, 62,1319-1341.

[16] O. Dahlman. Seismic source and transmission functions from underground nuclear explosions. Bull.Seismol.Soc.Am. 1974, 64,1275-1293.

[17] T. Lay, D.V. Helmberger and D.G. Harkrider. Source models and yield-scaling relations for underground nuclear explosions at Amchitka Island. Bull.Seismol.Soc.Am. 1984, 74,843-862.

[18] A.S. Kiremidjian and T. Anagnos. Stochastic slip predictable models for earthquake occurrences. Bull.Seismol.Soc.Am. 1984, 74,739-755.

[19] R.A. Harris and R.W. Simpson. Changes in static stress on southern California faults after the 1992 Landers earthquake. Nature. 1992, 360,251-254.

[20] R.A. Harris, R.W. Simpson and P.A. Reasenberg. Influence of static stress changes on earthquake locations in southern California. Nature. 1995, 375,221-224.

[21] R.A. Harris and R.W. Simpson. In the shadow of 1857-The effect of the great Ft. Tejon earthquake on subsequent earthquakes in southern California. Geophys. Res. Lett. 1996, 23,229-232.

[22] W. Marzocchi, L. Sandri and E. Boschi. On the validation of earthquake-forecasting models: The case of pattern recognition algorithms. Bull.Seismol.Soc.Am. 2003, 93,1994-2004.

[23] A. Helmstetter, Y.Y. Kagan and D.D. Jackson. Comparison of short-term and long-term earthquake forecast models for Southern California. Bull.Seismol.Soc.Am. 2006, 96,90-106.

[24] Yi-Fang Chang. Nonlinear dynamics, magnitude-period formula and new research on earthquake. International Journal of Sciences. 2012, Nov. 1-9.

[25] G.L. Vasconcelos. First-Order Phase Transition in a Model for Earthquakes. Phys. Rev. Lett. 1996, 76,4865-4867.

[26] D.L. Turcotte. Fractals in geology and geophysics. Pure Appl.Geophys. 1989,131,171-196.

[27] M. Bath. Introduction to Seismology. Birkhauser Verlag. 1979.

[28] I.Tsubokawa. On relation between duration of crustal movement and earthquake magnitude. J. Geod. Soc. Japan. 1969, 15,7588

[29] C.H. Scholz, L.R. Sykes and Y.P. Aggarwal. Earthquake prediction: a physical basis. Science. 1973, 181,803-809.

[30] Yi-Fang Chang, Topological physics, topological sciences and new research of string. International Journal of Modern Mathematical Sciences. 2015,13(1):86-100.

[31] S.W. Smith and W. Van de Lindt. Strain adjustments associated with earthquakes in southern California. Bull.Seismol.Soc.Am. 1969, 59,1569-1589.

[32] R.L. Kovach. Source mechanisms for Wilmington oil field, California, subsidence earthquakes. Bull.Seismol.Soc.Am. 1974, 64,699-711.

[33] G.M. Mavko, S. Schulz and B.D. Brown. Effects of the 1983 Coalinga, California, earthquake on creep along the San Andreas fault. Bull.Seismol.Soc.Am.1985, 75,475-489.

[34] D.H. Oppenheimer, P.A. Reasenberg and R.W. Simpson. Fault plane solutions for the 1984 Morgan Hill, California, earthquake sequence: Evidence for the state of stress on the Calaveras fault. J.Geophys.Res. 1988, 93,9007-9026.

[35] P.A. Reasenberg and L.M. Jones. Earthquake hazard after a main shock in California. Science. 1989, 243:1173-1176.

[36] S.C. Jaumé and L.R. Sykes. Change in the state of stress on the southern San Andreas fault resulting from the California earthquake sequence of April to June 1992. Science. 1992, 258,1325-1328.

[37] R.S. Stein, G.C.P. King and J.Lin. Stress triggering of the $1994 \mathrm{M}=6.7$ Northridge, California, earthquake by its predecessors. Science. 1994, 265,1432-1435.

[38] D.A. Dodge, G.C. Beroza and W.L. Ellsworth. Detailed observations of California foreshock sequences: Implications for the earthquake initiation process. J.Geophys.Res. 1996, 101,22371-22392.

[39] J. Deng and L.R. Sykes. Evolution of the stress field in southern California and triggering of moderate-size earthquakes: A 200year perspective. J.Geophys.Res. 1997, 102,9859-9886.

[40] J. Deng and L.R. Sykes. Stress evolution in southern California and triggering of moderate-, small-, and micro-size earthquakes. J.Geophys.Res. 1997, 102,24411-24435.

[41] Y. Ogata, L. Jones and S. Toda. When and where the aftershock activity was depressed: Contrasting decay patterns of the proximate large earthquakes in southern California. J.Geophys.Res. 2003, 108 (B6):2318.

[42] M.C. Gerstenberger, S. Wiemer, L.M. Jones, et al. Real-time forecasts of tomorrow's earthquakes in California. Nature. 2005, 435,328-331.

[43] J.B. Ebel, D.W. Chambers, A.L. Kafka and J.A. Baglivo. Non-Poissonian earthquake clustering and the hidden Markov model as bases for earthquake forecasting in California. Seismol. Res. Lett. 2007, 78,57-65.

[44] Yi-Fang Chang, Three ways of earthquake prediction. J.Relig.Psych.Res. 2004,27(3):126-130.

[45] Yi-Fang Chang, Chaos, magnitude-period formula of earthquakes and their multilevel prediction. World Institute for Scientific Exploration (WISE) Journal. 2017,6(2):51-59.

[46] E.N. Lorenz. Deterministic nonperiodic flow. J.Atmos.Sci. 1963, 20,130-141. 
[47] D.P. Robinson, C. Henry, S. Das and J.H. Woodhouse. Simultaneous rupture along two conjugate planes of the Wharton Basin earthquake. Science. 2001, 292,1145-1148.

[48] M.C. Gerstenberger, S. Wiemer, L.M. Jones and P.A. Reasenberg. Real-time forecasting of tomorrow's earthquakes in California. Nature. 2005, 435,328-331.

[49] O. Ramos, E. Altshuler and K.J. Måløy. Quasiperiodic events in an earthquake model. Phys.Rev. Lett. $2006,96(9): 098501$.

[50] Z.Y. Tao and Y. Ji. Special functions and earthquake prediction. Qigong and Science. 1990, 3:7-8.

[51] J.L. Kirschvink. Earthquake prediction by animals: evolution and sensory perception. Bull.Seismol.Soc.Am. 2000, 90,312-323. 